\title{
The utility of PET-CT in the staging and management of advanced and recurrent malignant melanoma
}

\author{
SH Twycross' ${ }^{1}$, H Burger ${ }^{1}$, J Holness ${ }^{2}$ \\ ${ }^{1}$ Division of Radiation Oncology, Department of Medical Imaging and Clinical Oncology, Stellenbosch University \\ and Tygerberg Academic Hospital, Cape Town, South Africa \\ ${ }^{2}$ Division of Nuclear Medicine, Department of Medical Imaging and Clinical Oncology, Stellenbosch University \\ and Tygerberg Academic Hospital, Cape Town, South Africa
}

Corresponding author: Sarah Twycross (sarah.twycross@gmail.com)

\begin{abstract}
Background: Accurate pre-operative staging and correct surgical selection of patients with malignant melanoma reduces unnecessary morbidity and mortality, improves distant control and may improve survival. ${ }^{18} \mathrm{~F}$-fluorodeoxyglucose Positron Emission Tomography Computed Tomography $\left({ }^{18} \mathrm{~F}-\mathrm{FDG}\right.$ PET-CT) has been shown to be useful in exclusion of metastatic sites and aids in surgical planning in stage III and potentially resectable stage IV disease. The primary objective of the study was to determine whether the use of PET-CT alters the initial staging and management of patients with advanced and recurrent melanoma.

Methods: Retrospective analysis of clinical records of patients with malignant melanoma referred for staging PET-CT over a three-year period at our institution was performed. Pre- and post-PET-CT stage was recorded and a descriptive analysis was done to determine whether PET-CT resulted in a change in stage grouping and whether this change effected a change in clinical management.

Results: A change in stage grouping occurred in $21 / 39$ (53.8\%) of patients, $76.2 \%$ of which were up-staged and $23.8 \%$ down staged. On analysis of stage III/IV and recurrent melanoma, a change in stage occurred in $90 \%$ of stage III, $50 \%$ of stage IV and $50 \%$ of recurrent melanoma patients. This effected a change in management in $86.7 \%$ of patients with stage III, IV and recurrent melanoma collectively.

Conclusion: PET-CT is a useful tool in the staging and subsequent management of melanoma. Its utility is pronounced in advanced and recurrent melanoma.
\end{abstract}

S Afr J Surg 2019;57(3)

http://dx.doi.org/10.17159/2078-5151/2019/v57n3a2832

\section{Introduction}

Cutaneous melanoma is the most common cause of skin cancer mortality. ${ }^{1}$ Although only $5 \%$ of skin cancers are melanomas, it accounts for more than $50 \%$ of skin cancer deaths. ${ }^{2}$ There is a rising incidence of melanoma reported worldwide. ${ }^{1-4} \mathrm{~A}$ study in Cape Town identified its white sub-population as having amongst the highest incidence in the world for primary melanoma. ${ }^{3}$

Primary melanoma develops most commonly on the skin and its pattern of spread is usually via lymphatics to regional lymph nodes or via haematogenous spread to distant metastatic sites including lung, liver, brain, peripheral nerves, skin and soft tissue. ${ }^{4}$

Advanced melanoma includes American Joint Committee on Cancer (AJCC) $7^{\text {th }}$ Edition ${ }^{5}$ stage III and IV and encompasses melanoma with nodal or distant spread. It is associated with a particularly poor prognosis, and early diagnosis and accurate staging affect prognosis and survival. ${ }^{1,6}$
In advanced melanoma, surgery is the most effective means of achieving loco-regional control. ${ }^{7}$ It remains the standard approach for patients with regional nodal metastases, provided it does not lead to unacceptable morbidity and mortality. In selected cases, surgery can provide distant control and metastasectomy has been associated with improved overall survival in stage IV melanoma. ${ }^{4,6-8}$

Accurate preoperative staging is essential to ascertain which patients may benefit from further surgery after initial diagnostic biopsy. ${ }^{4,7}$ It allows for subsequent surgical control of limited loco-regional disease and avoids the morbidity of lymph node dissection in inappropriately selected patients, including those with unresectable loco-regional disease or occult distant metastases. ${ }^{4}$

PET-CT has been shown to exclude other sites of metastatic disease and aid in surgical planning both in stage III and potentially resectable stage IV disease. ${ }^{2}$ Current guidelines endorse the use of PET-CT staging in stage III and IV melanoma and do not recommend PET-CT staging in early melanoma (stage I and II). ${ }^{9}$ 
The diagnostic accuracy and the value of PET is highest in higher stages of melanoma, in the setting of macroscopic nodal disease (stage IIIB and higher). ${ }^{6,10}$ In a systematic review, PET sensitivity has been reported to be $68-87 \%$ for stage III and IV melanoma compared to $0-67 \%$ for stage I and II melanoma. ${ }^{10}$ In a small study of 33 patients by Horn et al., $12 \%$ of patients had true melanoma metastases identified on PET, none of which were identified by conventional imaging. The PET findings upgraded staging in these patients from stage III to IV. ${ }^{1,11}$

In our resource-limited setting, the obligation exists for judicious use of PET-CT directed by both comprehensive assessment of its utility in preoperative staging, and assessment of the effect of its use on clinical management decisions. Clear guidelines for its use are necessary to facilitate appropriate evidence-based and context appropriate care via the multidisciplinary team.

The primary objective of this study was to determine whether PET-CT alters staging and management in advanced and recurrent melanoma.

\section{Methods}

A retrospective analysis of clinical records of patients with malignant melanoma referred for staging PET-CT over a three-year period from February 2012 to February 2015 was performed. Patients included in the analysis were those with histologically confirmed cutaneous malignant melanoma and clinically evident Stage I-IV or recurrent disease referred for PET-CT at The Western Cape Academic PET-CT Centre, Cape Town, South Africa. Patients referred for PET-CT from health care institutions other than our institution were excluded from the analysis, due to unavailability of post-PETCT clinical records. Further exclusions included patients with an additional primary cancer other than melanoma or basal cell carcinoma (diagnosed within five years after melanoma diagnosis), patients younger than 18 years of age, choroidal or conjunctival melanoma, and those with incomplete clinical records.

The study was conducted with the approval of the Stellenbosch University Human Research Ethics Committee, as well as the institutional review board of the hospital (HREC reference number S15/10/252).

Sampling was conducted with the permission and use of records from the Western Cape Academic PET-CT Centre database. Data from the clinical records of the identified referrals was reviewed with the aid of electronic databases, including the National Health Laboratory System (NHLS), the Tygerberg Hospital Enterprise Content Management (ECM) System and the Picture Archiving and Communication System (PACS). Where electronic data was not available, hard copy clinical records were reviewed. Patient characteristics including age, gender, HIV status and serum lactase dehydrogenase (LDH) were recorded. Ethnicity, although important, was poorly recorded and hence not captured.

Tumour characteristics including tumour, nodal and metastasis (TNM) and AJCC staging were recorded.
Histological subtypes were grouped for analysis into nonacral lentiginous cutaneous, acral lentiginous cutaneous, mucosal and unknown subtype. Melanoma sites were grouped for analysis into trunk, limbs, head and neck, mucosal or nodal. Nodal sites were defined as pathologically confirmed melanoma in a lymph node, without a primary site identified. Baseline imaging included a combination of chest $\mathrm{X}$-ray (CXR) and ultrasound scan (USS) of the abdomen or CT scan of the chest and abdomen.

${ }^{18}$ F-FDG PET-CT scans were performed as per Tygerberg Hospital/Stellenbosch University nuclear medicine protocols, an adaptation of European Association of Nuclear Medicine (EANM) procedure guidelines. ${ }^{12}$ Scans were interpreted visually by an experienced nuclear medicine physician in consultation with a qualified radiologist. PET-CT reports with imaging findings interpreted as 'suspicious for' or 'consistent with' were considered positive for occult metastatic disease. This is consistent with studies performed elsewhere. ${ }^{8}$

Pre- and post-FDG PET-CT TNM staging was captured and analysed according to AJCC $7^{\text {th }}$ Edition staging. ${ }^{5}$ Tumour stage included Breslow thickness and the presence or absence of ulceration. AJCC stage groupings were used to categorise patients into pre- and post-FDG PET-CT stage groups. Recurrence (local and regional) was documented as a separate stage entity, irrespective of TNM stage or AJCC stage group. It was further documented whether PET-CT resulted in a change in stage grouping and whether the change in stage grouping effected a change in clinical management.

Stage III, IV and recurrent melanomas were analysed separately, in keeping with the primary objective of the study.

Simple descriptive analysis was performed.

\section{Results}

\section{Exclusions}

Of the 66 patients referred for FDG PET-CT at Tygerberg Hospital over the three-year period, 27 were excluded from the analysis. The study cohort therefore comprised 39 patients. Reasons for exclusion are documented in Table 1.

\begin{tabular}{|c|c|}
\hline Exclusion reason & n (\%) \\
\hline 1. Incomplete clinical records & $4(14.8)$ \\
\hline $\begin{array}{l}\text { 2. Patients not referred via the radiation } \\
\text { oncology dermatology MDT }\end{array}$ & $7(25.9)$ \\
\hline 3. Synchronous primary (non-BCC) & $5(18.5)$ \\
\hline 4. Previously captured in data-set & $2(7.4)$ \\
\hline 5. Not a melanoma primary & $3(11.1)$ \\
\hline 6. Lost to follow-up & $2(7.4)$ \\
\hline 7. Choroidal/conjunctival melanoma & $2(7.4)$ \\
\hline $\begin{array}{l}\text { 8. PET-CT not qualified as baseline staging } \\
\text { investigation }\end{array}$ & $2(7.4)$ \\
\hline TOTAL & $27(100)$ \\
\hline
\end{tabular}

MDT, multidisciplinary team; BCC, basal cell carcinoma 


\section{Patient characteristics}

Patient characteristics are shown in Table 2.

\begin{tabular}{lc} 
Table 2: Patient characteristics & \\
\hline Age & \\
Mean age (range) years & n (\%) \\
\hline Patient Characteristic & \\
\hline Gender & $20(51.3)$ \\
Male & $19(48.7)$ \\
Female & $39(100)$ \\
\hline TOTAL & \\
HIV status & $3(7.7)$ \\
Positive & $17(43.6)$ \\
Negative & $19(48.7)$ \\
Unknown & $39(100)$ \\
\hline TOTAL & \\
LDH & $5(12.8)$ \\
Normal & $18(46.2)$ \\
Raised & $16(41.0)$ \\
Unknown & $39(100)$ \\
\hline TOTAL &
\end{tabular}

\section{Tumour characteristics}

Twenty-three $(59.0 \%)$ of the analysed subset $(n=39)$ had melanoma of the limbs. The number of patients with truncal melanoma was eight $(20.5 \%)$, head and neck three $(7.7 \%)$, primary nodal three $(7.7 \%)$, and mucosal membranes two (5.1\%). Histological subtypes are shown in Table 3.

\begin{tabular}{lc} 
Table 3: Histological subtype & \\
\hline Histological subtype & $\mathbf{n}(\mathbf{\%})$ \\
\hline Acral lentiginous & $8(20.5)$ \\
Non-acral lentiginous & $4(10.3)$ \\
Mucosal & $2(5.1)$ \\
Unreported & $25(64.1)$ \\
\hline TOTAL & $39(100)$
\end{tabular}

Pre-PET-CT TNM and AJCC stage grouping are documented in Table 4. Baseline imaging used pre-PET-CT included a combination of CXR and USS of the abdomen or computed tomography (CT) scans of the chest and abdomen. This was not standardised in all patients. Six (15.4\%) patients had a combination of CXR and abdominal USS at baseline. According to previous protocols in the department this was the routine minimum investigation prior to clinical management decision making. Only two (5.1\%) patients had a baseline contrasted CT of the chest and abdomen.

Histopathologically confirmed T4 tumours accounted for 11 (28.2\%) of the 39 patients.

Prior to PET-CT $18(46.2 \%)$ patients were found to be $\mathrm{cN} 0$. One patient had negative sentinel lymph node biopsy
Table 4: Pre-PET-CT TNM and AJCC stage grouping

\begin{tabular}{|c|c|}
\hline \multicolumn{2}{|l|}{ Tumour characteristics: } \\
\hline Tumour T-stage $n=39$ & n (\%) \\
\hline Tis & $2(5.1)$ \\
\hline $\mathrm{T} 1$ & $2(5.1)$ \\
\hline $\mathrm{T} 2$ & $4(10.3)$ \\
\hline $\mathrm{T} 3$ & $3(7.7)$ \\
\hline $\mathrm{T} 4$ & $11(28.2)$ \\
\hline Unreported Breslow & $13(33.3)$ \\
\hline *Local recurrence & $4(10.3)$ \\
\hline a: no ulceration & $12(30.8)$ \\
\hline b: ulceration & $12(30.8)$ \\
\hline Unreported ulceration & $15(38.5)$ \\
\hline \multicolumn{2}{|l|}{ Nodal N-stage $n=39$} \\
\hline N0 & $18(46.2)$ \\
\hline N1a & $0(0.0)$ \\
\hline $\mathrm{N} 1 \mathrm{~b}$ & $1(2.6)$ \\
\hline $\mathrm{N} 2 \mathrm{a}$ & $0(0.0)$ \\
\hline $\mathrm{N} 2 \mathrm{~b}$ & $5(12.8)$ \\
\hline $\mathrm{N} 2 \mathrm{c}$ & $2(5.1)$ \\
\hline N3 & $8(20.5)$ \\
\hline *Nodal recurrence & $3(7.7)$ \\
\hline Unreported nodal status & $2(5.1)$ \\
\hline \multicolumn{2}{|l|}{ Metastasis M-stage $n=39$} \\
\hline M0 & $29(74.4)$ \\
\hline M1a & $0(0.0)$ \\
\hline M1b & $1(2.6)$ \\
\hline M1c & $7(17.9)$ \\
\hline Unreported metastatic status & $2(5.1)$ \\
\hline \multicolumn{2}{|l|}{ AJCC stage group $n=39$} \\
\hline 0 & $1(2.6)$ \\
\hline I & $4(10.3)$ \\
\hline II & $7(17.9)$ \\
\hline III & $10(25.6)$ \\
\hline IV & $8(20.5)$ \\
\hline Recurrence* & $4(10.3)$ \\
\hline Unreported stage group & $5(12.8)$ \\
\hline
\end{tabular}

* Recurrence is not part of TNM staging and in the study included local and nodal recurrences, but not metastatic progression

(SLNB). This can be ascribed to delay in referral for PETCT. Strict protocol for PET-CT was not in place at the time of referral.

Prior to PET-CT staging, one (2.6\%), four $(10.3 \%)$, seven $(17.9 \%)$, ten $(25.6 \%)$, and eight $(20.5 \%)$ patients accounted for stage 0 , I, II, III, and IV respectively. There were four $(10.3 \%)$ recurrences and five $(12.8 \%)$ of unknown stage group.

Post PET-CT stage grouping was as follows: two (5.1\%), five $(12.8 \%)$, six $(15.4 \%), 4(10.3 \%)$ and $18(46.2 \%)$ patients 
accounted for stage group 0, I, II, III and IV respectively. There were two $(5.1 \%)$ recorded recurrences and two $(5.1 \%)$ patients with unknown stage grouping.

\section{The effect of PET-CT on stage grouping (Table 5)}

In the entire cohort, $16(41.0 \%)$ patients were upstaged and $5(12.8 \%)$ down-staged after PET-CT staging. In stage 0 -II patients, a change in stage occurred in 3/12 (25.0\%) of patients. In the clinical stage III/IV and recurrence group, a change in stage occurred in $15 / 22(68.2 \%)$ patients. Ten $(45.5 \%)$ cases were upstaged, and five $(22.7 \%)$ down-staged in this group.

\begin{tabular}{lcccc}
\multicolumn{5}{l}{ Table 5. Change in stage with PET-CT } \\
\hline & Upstaged & $\begin{array}{c}\text { Down- } \\
\text { staged }\end{array}$ & Same & TOTAL \\
\hline Stage 0-II & $3(25 \%)$ & $\mathrm{n} / \mathrm{a}$ & $9(75 \%)$ & 12 \\
Stage III & $8(80 \%)$ & $1(10 \%)$ & $1(10 \%)$ & 10 \\
Stage IV & $\mathrm{n} / \mathrm{a}$ & $4(50 \%)$ & $4(50 \%)$ & 8 \\
Recurrence & $2(50 \%)$ & 0 & $2(50 \%)$ & 4 \\
Unknown & $3(60 \%)$ & 0 & $2(40 \%)$ & 5 \\
\hline TOTAL & $16(41.0 \%)$ & $5(12.8 \%)$ & $18(46.2 \%)$ & $39(100 \%)$
\end{tabular}

n/a, not applicable

\section{The effect of PET-CT in changing management}

Of those with an altered stage in the III/IV and recurrence group $(n=15), 13(86.7 \%)$ had a change in management. Definitive surgery was cancelled in six $(46.1 \%)$ patients and the management plans of four $(30.8 \%)$ patients now included surgery. This had not been the plan prior to PET-CT. One of these four patients had melanoma of the back with clinically palpable axillary lymph nodes and subcutaneous nodules. PET-CT excluded disseminated subcutaneous nodules and the patient was down-staged from stage IV to IIIc and went on to have a wide local excision (WLE) and axillary lymph node dissection (ALND). The second patient had melanoma of the left upper arm with bilateral axillary lymph nodes palpable and a raised LDH. PET-CT excluded the lymphadenopathy and the patient was down-staged from stage IV to IIB, with a subsequent plan for WLE and SLNB. A third patient had melanoma of the left forearm with bilateral axillary lymph nodes and a raised LDH. PET-CT excluded lymphadenopathy and downstaging from stage IV to IIB led to a WLE and ALND. Notably SLNB is current protocol for this stage. The fourth patient had an unknown Breslow thickness but clinically palpable lymphadenopathy, satellite lesions and a suspicious lung lesion. Regional and distant metastatic spread were excluded with PET-CT and the patient was downstaged from stage IV to N0M0 (Stage I-II) disease and WLE and SLNB were performed. Other changes in management included cancellation of lymph node dissection in one (7.7\%) patient, further investigations performed prior to further management in one patient and in one patient surgery was declined.

\section{Discussion}

Our study suggests that staging PET-CT is responsible for a change in stage in the majority of melanoma cases. In keeping with other studies, the effect is more pronounced in advanced melanoma. ${ }^{2,10,13-15}$ PET-CT staging in this group is endorsed by current South African guidelines. ${ }^{9}$

A change in AJCC stage group with PET-CT was detected in $53.8 \%$ of the entire group of patients and in $68.2 \%$ when the stage III/IV/recurrence group was analysed separately. Of those who had a change in stage in this sub-group, a large proportion $(86.7 \%)$ had a management change.

Controversy exists as to the utility of PET as part of preoperative staging in early stage melanoma. ${ }^{2,14,16,17}$ There is little data to support the routine use of PET over conventional imaging such as chest $\mathrm{x}$-ray (CXR) and computed tomography (CT) in all stages of melanoma. Most studies show low sensitivity in detecting distant disease in patients with clinically localized disease and PET is not routinely indicated for early stage disease. ${ }^{2,13-15}$ To date it has been shown that PET-CT has a limited role in N0 disease. In patients without clinical evidence of nodal metastases, SLNB is the gold standard staging procedure. It is associated with high sensitivity and specificity, limited morbidity and high prognostic significance. . $^{4,13,18}$

In this study 12 patients with N0M0 (stage 0-II) disease were referred for PET-CT and only $5(41.7 \%)$ of these had T4 disease. One was referred for PET-CT despite negative SLNB, which is the staging procedure of choice in N0 disease. It is the policy of the study institution to limit referral for PETCT to patients with stage III/IV and recurrent melanoma and referral of clinically node negative non-metastatic cases may be viewed as inappropriate allocation of resources. Only three of 12 stage $(25 \%)$ N0M0 referrals were upstaged, two of which were T4. Two were upstaged from stage II to III but one was upstaged from stage II (T4bN0M0) to IV (T4bN3M1c). The study results confirm the validity of our current protocol and the high proportion of patients referred for PET-CT with stage 0-II disease reflects a need for centralised discussion and multidisciplinary review prior to further staging investigations.

Although survival outcomes were not analysed in this study, previous literature suggests that in clinically stage III disease PET-CT positivity is independently predictive of worse melanoma specific survival. ${ }^{17}$ A survival benefit is suggested with PET-CT staging and Dalrymple-Hay et al. published improved survival rates thought to be due to improved patient selection for surgery. ${ }^{19,20}$ In an advancing era of systemic targeted and immunotherapy, it may be considered that this benefit is underestimated and the need for accurate staging in advanced and recurrent disease is pivotal to outcome. In the South African public health setting it may be considered extravagant to direct resources towards expensive staging investigations such as PET-CT. However, immunotherapy is unlikely to be accessible in such settings and it remains paramount that oligometastatic patients amenable to metastasectomy be found with the aid of PET-CT. 
A major limitation of this study was its retrospective nature. This poses the risk of selection bias, especially in the absence of a mitigating multivariate analysis, which was not possible due to small numbers. Prospective trials are lacking and further evidence is needed to justify the routine use of PET-CT over conventional imaging. Of note in our study, comparative crosssectional imaging was not standard and it was not possible to conclude the absolute benefit of PET-CT over conventional CT imaging. Available studies do however suggest that PETCT is more sensitive and specific than conventional CT. ${ }^{19,21}$ Departmental protocols should be individualised according to resources. PET-CT is an expensive imaging tool, however in light of the cost-effectiveness of facilitating appropriate management in advanced and recurrent melanoma, its use may be justified.

A high number of exclusions resulted in fewer cases amenable to analysis which limited the power of our study. This was in part due to incomplete records and patients lost to follow-up. Poorly completed baseline records precluded satisfactory data collection to draw conclusions on the association of individual histopathological factors and a change in stage. Patient characteristics including ethnicity, HIV status and LDH levels were poorly recorded in clinical records. These are noteworthy omissions, given that ethnicity influences the incidence of melanoma, ${ }^{22}$ and HIV status influences the specificity of PET-CT findings. ${ }^{23} \mathrm{LDH}$ levels are important to stage M1 disease according to AJCC $7^{\text {th }}$ Ed. staging. Our findings are useful to direct accurate future clinical record keeping which would allow future research in our unique clinical setting. Further limitation was that PETCT findings confirming upstaging or down-staging were not correlated with pathological findings.

Future directions as to whether Breslow depth and the presence or absence of ulceration justifies staging with PET$\mathrm{CT}$ in node negative disease and stratifying results for HIV positive individuals are necessary. A correlation analysis would aid in defining the individual risk of a change in stage and management.

\section{Conclusion}

The results of our study favour the utility of PET-CT in the staging of advanced and recurrent melanoma. In this study a change in stage occurred in just over half of the entire cohort, and in $68.2 \%$ of the advanced and recurrent melanoma subset. PET-CT may effect a change in stage with clinical consequences, which was the case in $86.7 \%$ of patients with advanced and recurrent melanoma. It is our recommendation that PET-CT should be used as baseline imaging for stage III, IV and recurrent melanoma, which is in keeping with current guidelines. This remains relevant in resource limited settings, taking into account that accurate imaging effects changes in management. Appropriate selection of patients for PET-CT should take place within the context of a multidisciplinary team to ensure appropriate evidence-based care. It is clear that rigorous record keeping and adherence to clinical guidelines should be advocated in order to ensure optimal management and strengthen further research.

\section{Acknowledgements}

We thank $\mathrm{Mr}$ Michael McCaul from the Division of Epidemiology and Biostatistics at the University of Stellenbosch for assistance with statistical analysis. We thank Dr Hannelie Vermeulen at the Department of Radiation Oncology Tygerberg Hospital for assistance in data collection.

\section{Conflict of interest}

The authors have no conflict of interest to declare.

\section{Author contributions}

The first author was responsible for the conception, design, analysis and interpretation of the data as well as the drafting and review of the manuscript. The second and third authors supervised the design, analysis and interpretation of the data and critically reviewed and approved the final manuscript.

\section{Funding sources}

None

\section{REFERENCES}

1. Constantinidou A, Hofman M, O'Doherty M, Acland KM, Healy C, Harries M. Routine positron emission tomography and positron emission tomography/computed tomography in melanoma staging with positive sentinel node biopsy is of limited benefit. Melanoma Res. 2008;18(1):56-60.

2. Choi EA, Gershenwald JE. Imaging studies in patients with melanoma. Surg Oncol Clin N Am. 2007;16(2):403-30.

3. Jessop S, Stubbings H, Sayed R, Duncan-Smith J, Schneider JW, Jordaan HF. Regional clinical registry data show increased incidence of cutaneous melanoma in Cape Town. S Afr Med J. 2008;98(3):197-9.

4. Patnana M, Bronstein Y, Szklaruk J, et al. Multimethod imaging, staging, and spectrum of manifestations of metastatic melanoma. Clin Radiol. 2011;66(3):224-36.

5. Balch CM, Gershenwald JE, Soong SJ, et al. Final version of 2009 AJCC melanoma staging and classification. J Clin Oncol. 2009;27: 6199-206. : í

6. Bronstein Y, Ng CS, Rohren E, Ross MI, et al. PET/CT in the management of patients with stage IIIC and IV metastatic melanoma considered candidates for surgery: evaluation of the additive value after conventional imaging. AJR Am J Roentgenol. 2012;198(4):902-8.

7. Angel M, Rodriguez R, Alabbas H, Ramjaun A, Meguerditchian A. Value of positron emission tomography scan in stage III cutaneous melanoma: A systemic review and meta-analysis. Surg Onco. 2014;23:11-6.

8. Brady MS, Akhurst T, Spanknebel K, et al. Utility of preoperative [(18)] f fluorodeoxyglucose-positron emission tomography scanning in high-risk melanoma patients. Ann Surg Oncol. 2006;13(4):525-32.

9. Vorster M, Doruyter A, Brink A, et al. Appropriate indications for positron emission tomography/computed tomography, 2015. SAMJ. 2016;106(1):112.

10. Schroer-Günther MA, Wolff RF, Westwood ME, et al. F-18fluoro-2- deoxyglucose positron emission tomography (PET) and PET/computed tomography imaging in primary staging of patients with malignant melanoma: a systematic review. Syst Rev. 2012;1(62):1-11.

11. Horn J, Lock-Andersen J, Sjostrand H, Loft A. Routine use of FDG-PET scans in melanoma patients with positive sentinel node biopsy. Eur J Nucl Med Mol Imaging. 2006;33(8):887-92. 
12. Boellaard R, O'Doherty MJ, Weber WA, et al. FDG PET and PET/CT: EANM procedure guidelines for tumour PET imaging: version 1.0. Eur J Nucl Med Mol Imaging. 2009. doi 10.1007/ s00259-009-1297-4

13. Maubec E, Lumbroso J, Masson F, et al. F-18 fluorodeoxyD-glucose positron emission tomography scan in the initial evaluation of patients with a primary melanoma thicker than 4 mm. Melanoma Res. 2007;17(3):147-54.

14. Wagner T, Chevreau C, Meyer N, Mourey L, Courbon F, Zerdoud S. Routine FDG PET -CT in patients with a highrisk localized melanoma has a high predictive positive value for nodal disease and high negative predictive value for the presence of distant metastases. J Eur Acad Dermatol Venereol. 2012;26(11):1431-35.

15. Clark PB, Soo V, Kraas J, Shen P, Levine EA. Futility of fluorodeoxyglucose $\mathrm{F} 18$ positron emission tomography in initial evaluation of patients with T2 to T4 melanoma. Arch Surg. 2006;141(3):284 -8.

16. McIvor J, Siew T, Campbell A, McCarthy M. FDG PET in early stage cutaneous malignant melanoma. J Med Imaging Radiat Oncol. 2014;58(2):149-54; quiz 266.

17. Niebling MG, Bastiaannet E, Hoekstra OS, Bonenkamp JJ, Koelemij R, Hoekstra HJ. Outcome of clinical stage III melanoma patients with FDG-PET and whole-body CT added to the diagnostic workup. Ann Surg Oncol. 2013;20(9):3098105 .

18. Morton DL, Thompson JF, Cochran AJ, et al. Final trial report of sentinel-node biopsy versus nodal observation in melanoma. N Eng J Med. 2014;370(7):599-609.

19. Schüle S-C, Eigentler TK, Garbe C, la Fougère C, Nikolaou $\mathrm{K}$, Pfannenberg C. Influence of 18F-FDG PET-CT on therapy management in patients with stage III/IV malignant melanoma. Eur J Nucl Mol Imaging. 2016;43:482-88.

20. Dalrymple-Hay MJR, Rome PD, Kennedy C, Fullham M, Cuaghan BC. Pulmonary metastatic melanoma - the survival benefit of positron emission tomography scanning, Eur J Cardiothoac Surg. 2002;21(4):611-5.

21. Xing Y, Bronstein Y, Ross MI, et al. Contemporary diagnostic imaging modalities for the staging and surveillance of melanoma patients: a meta-analysis. J Natl Cancer Inst. 2011;103(2):12942.

22. York K, Dlova NC, Wright CY, et al. Primary cutaneous malignancies in the Northern Cape Province of South Africa: A retrospective histopathological review. SAMJ. 2017;107(1):838.

23. Sathekge M, Maes A, Van de Wiele C. FDG-PET imaging in HIV infection and tuberculosis. Semin Nucl Med. 2013;43(5):349-66. doi:10.1053/j.semnuclmed.2013.04.008 